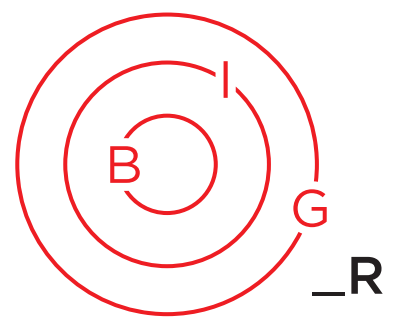

\section{ART \& BORDERS}

En français

\title{
Frontières \& Mythologies personnelles : un entretien avec Emeric Lhuisset
}

\author{
Elisa Ganivet *
}

Connu pour son travail de terrain dans les zones de conflit, le photographe Emeric Lhuisset a été interviewé par Elisa Ganivet. En se concentrant sur les projets contextuels de l'artiste, ses expériences in situ sont révélatrices de dynamiques territoriales singulières. Des extraits de l'entretien sont reproduits ici, dans la section spéciale Art \& Borders de cette édition La version anglaise a été publiée dans notre précédent numéro de BIG_Review (ici).

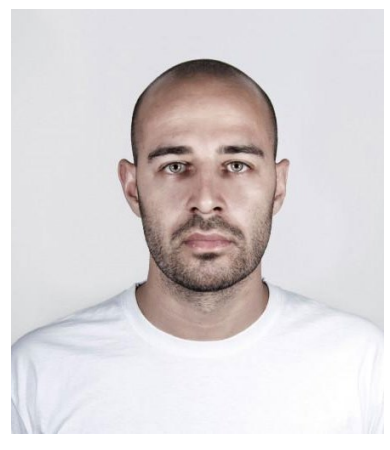

www.emericlhuisset.com

\section{A propos de l'artiste}

Né en 1983, Emeric Lhuisset a grandi en banlieue parisienne. Diplômé en art (Ecole des Beaux-Arts de Paris) et en géopolitique (Ecole Normale Supérieure Ulm - Centre de géostratégie / Université Paris 1 Panthéon-Sorbonne). Son travail est présenté dans de nombreuses expositions à travers le monde (Tate Modern à Londres, Museum Folkwang à Essen, Institut du Monde Arabe à Paris, Frac Alsace, Stedelijk Museum à Amsterdam, Rencontres d'Arles, Sursock Museum à Beyrouth, CRAC Languedoc-Roussillon, Musée du Louvre Lens...). Récemment il remporte la Résidence BMW pour la Photographie 2018 et Grand Prix Images Vevey - Leica Prize 2017. II a également été nominé notamment pour le Photographic Museum of Humanity Grant 2018 (Honorable Mention), pour le prix Coal (2016), pour le prix Magnum Foundation Emergency Fund (2015), pour le prix Niépce (2015), pour le Leica Oskar Barnack Award (2014) ainsi que pour le Prix HSBC pour la photographie (2014). II publie chez André Frère Editions et Paradox (Ydoc) Maydan - Hundred portraits (2014), Last water war (2016), chez André Frère Editions et Al-Muthanna L'autre rive (2017) et aux Editions Trocadero Quand les nuages parleront. Son travail est présent dans de nombreuses collections privées ainsi que dans celles du Stedelijk Museum, du Musée Nicéphore Niepce et du Musée de l'Armée - Invalides. En parallèle de sa pratique artistique, il enseigne à l'IEP de Paris (Sciences Po) sur la thématique art contemporain \& géopolitique. II est représenté par Kalfayan Galleries.

\footnotetext{
* Elisa Ganivet, doctor of philosophy, art historian, author of Border Wall Aesthetics (Columbia University Press 2020), has worked as a cultural manager and curator for international public and private cultural organizations. Web: www.ElisaGanivet.com
} 


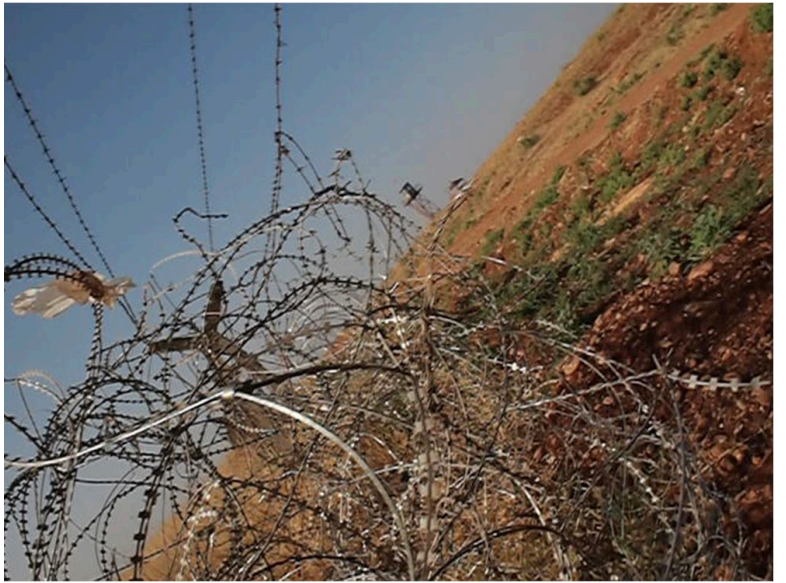

Figure 1. Passage de frontière, Syrie-Turquie, video still, 2010-2018. (c) Emeric Lhuisset. Courtoisie de l'artiste.

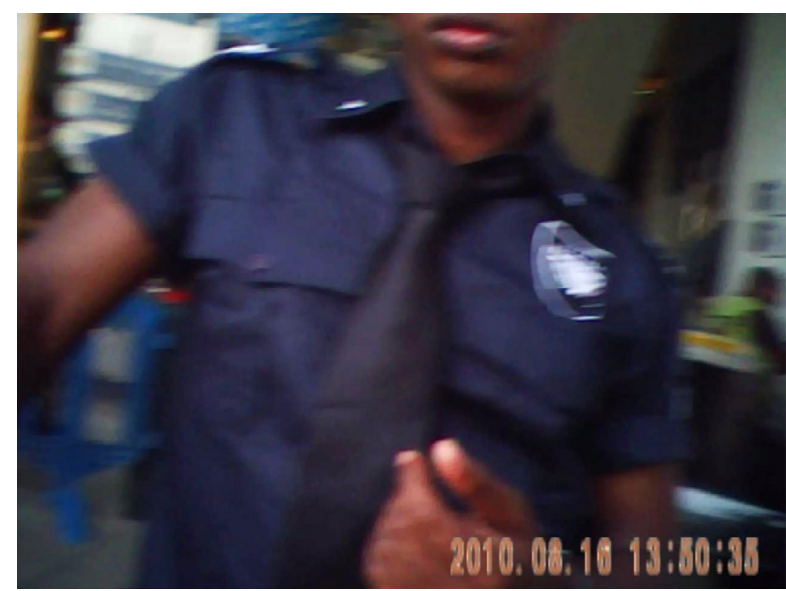

Figure 2. Voyage, archive photographique personnelle, 2010. (c) Emeric Lhuisset. Courtoisie de l'artiste.

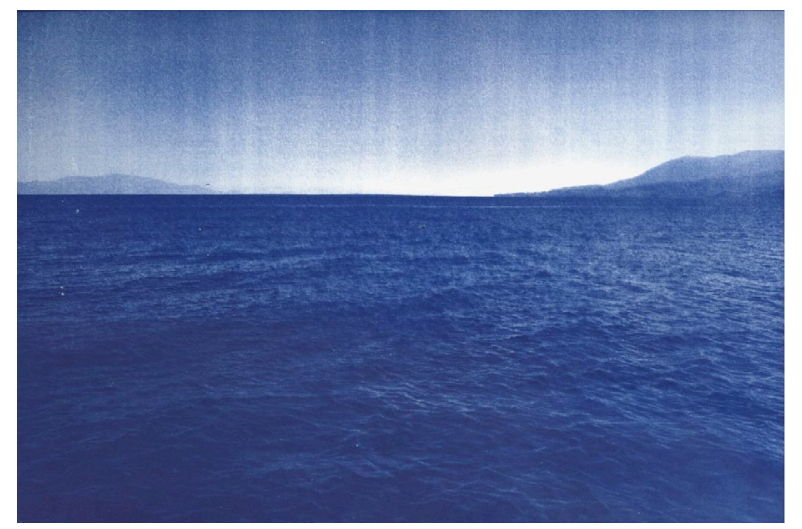

Figure 3. L'autre rive, Irak, Syrie, Turquie Grèce, Allemagne, Danemark, France, 2011-2017. (c) Emeric Lhuisset. Courtoisie de l'artiste.
Elisa Ganivet : Emeric, tu es un artiste internationalement reconnu pour tes recherches de terrain en zones de conflit, essentiellement menées au Moyen-Orient, tout autant qu'en Ukraine, en Colombie... La photographie est ton outil de prédilection et on t'en découvre d'autres au fil des années. Lorsqu'en préambule nous avions émis ensemble cette notion de la frontière, ta première remarque fut de souligner le fait que la guerre et la frontière étaient inextricablement liées. Dans ton corpus d'œuvres, il y en a-t-elles qui font explicitement référence à cette idée, à cette sensation de frontière ? Je pense en particulier à ton film Quand les Nuages parleront où à la fin on te suit, en caméra portée, dans l'un de tes franchissements clandestins, l'un des nombreux qui tu as pu faire entre la Syrie et la Turquie. Cette vidéo s'inscrit-elle dans une pratique spontanée ou bien dans une vision de les regrouper ultérieurement?

Emeric Lhuisset : Non pas spécialement mais je me suis toujours intéressé à cette idée de frontière, aussi bien à celle étatique que celle que l'on peut avoir au sein même d'un état. Par exemple sur le territoire israélien, (sans même parler du palestinien), il y a des endroits qui sont complétement morcelés. Déjà en 2010, les hangars pour prendre les bus s'apparentait à des aéroports avec des portiques, des scanners etc. Tu devais passer des barrières pour entrer dans un marché ou ensuite tes sacs étaient fouillés. En France aujourd'hui, on remarque que le dispositif sécuritaire s'est normalisé mais ce n'était pas du tout le cas à l'époque. Les nombreux checkpoints que j'ai pu passer lorsque j'ai travaillé en Irak, en Afghanistan, en Syrie, en Turquie ou même en Colombie, sont toujours une épreuve lourde et compliquée. Jusqu'au dernier moment, on ignore si on y parviendra, si les laisser-passer sont conformes aux attentes. II faut parfois ruser ce qui peut être oppressant. L'expérience est identique au Pakistan, un visa classique ne suffit pas pour passer des check points dans les zones tribales. En Irak, alors que j'avais un simple visa pour les zones kurdes, je passais des check points pour entrer dans les zones arabes. Donc il ne s'agit pas forcément de frontières purement étatiques mais d'un morcellement de l'espace. Cette sensation m'a toujours marqué. II y a aussi le cas des frontières qui ne sont pas visibles à l'œil nue. C'est le cas dans la forêt amazonienne par exemple où l'on traverse de la Colombie au Brésil sans le savoir. Cela m'a d'ailleurs causé quelques problèmes.

Ces vidéos réalisées, entre la Syrie et la Turquie sont des archives personnelles. Je documente régulièrement mes projets, mais ces archives, ne sont initialement pas destinées à être montrées [Figures 1 \& 2]. J'ai finalement décidé d'inclure cette séquence du passage de la Syrie vers la Turquie dans un projet sur les réfugiés au long court (2010-2018) et plus global. Ce qui m’a intéressé est ce moment précis « du devenir réfugié » lorsque les personnes qui fuient la guerre en Syrie franchissent les fils de fer barbelés 

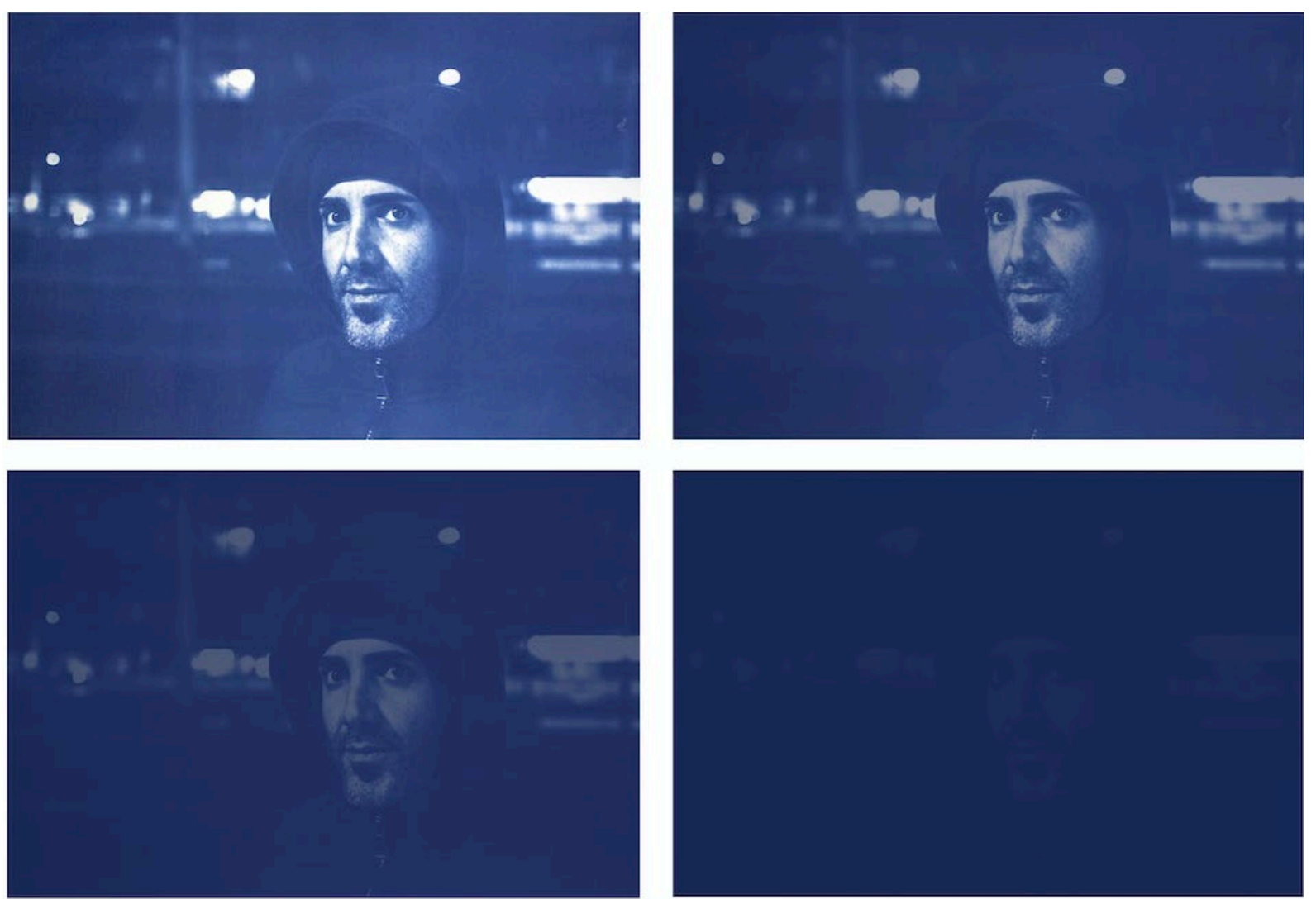

Figure 4. @ Emeric Lhuisset. L’autre rive, Irak, Syrie, Turquie Grèce, Allemagne, Danemark, France, 2011-2017. Courtoisie de l'artiste.

qui séparent ces des deux états. II s'agit d'un instant. Quelques centimètres avant, ils ne le sont pas encore, quelques centimètres après oui.

Inscrire cette séquence dans ce projet est un révélateur car j'aborde un préambule et un destin. C'est l'histoire de ces personnes avant d' "être réfugiées», celles qui le sont devenues ou qui auraient pu le devenir. Est également abordé le thème du franchissement : celui de la traversée en mer, celui de la traversée de l'Europe, puis de l'arrivée jusqu'à un territoire où ces personnes vont se fixer Avec le projet L'autre rive (2011-2017) [Figures 3 \& 4] ce qui m'intéresse c'est de parler de ces amis rencontrés en Irak ou en Syrie, devenus réfugiés, que j'ai retrouvé huit ans plus tard en Europe.

Dans mon livre intitulé Souvenirs de Syrie (2012) [Figures 5 \& 6], j'avais déjà réutilisé des archives personnelles. C'est lorsque je vivais avec des combattants rebelles syriens, caché dans une grotte dans la montagne que des photographies de ce quotidien très étranges avaient été prises. Un quotidien fait de bombardements, de tirs mais aussi d'attente et de tension. Initialement, je voulais le documenter pour mes propres archives. Mais un an après, alors que je regardais de nouveau ces images car l'un de mes amis avaient été tué et que je voulais me le remémorer par ce biais, je me suis dit qu'il fallait montrer ces photographies car elles étaient importantes, qu'elles témoignaient d'une réalité ignorée par beaucoup. La question qui se posa alors fut de comment les montrer car il ne s'agissait pas d'œuvres conçues comme telles. De plus, ces images étaient parfois très dures, assez violentes. On y voyait des gens torturés, des maisons détruites suite à un bombardement et toutes les atrocités qui accompagnent... Pour moi, accrocher ces images à un mur n'avait pas de sens, la tournure était obscène. J'avais aussi songé les donner à la presse.

EG : Mais ce geste aurait été à l'encontre de ta démarche artistique.

EL : L’idée c'était de rendre visible ces images, ce quotidien. Il était pour moi primordial de témoigner. Si la presse s'en emparait, il fallait savoir de quelle manière et en choisir les modalités. Mais comme ces images étaient déjà trop vieilles, elles avaient un an, leur obsolescence en terme journalistique m'a interpellé. II semble que même une image d'une semaine soit déjà dépassée. Cette dimension est un peu absurde car pour illustrer un article d'un homme en Syrie en train de tirer du haut de sa fenêtre, que cette photographie aie deux jours ou cinq ans, l'action reste la même. Vouloir qu'une photographie soit la plus fraîche, la plus instantanée possible est un peu absurde, dans ces situations. Du fait de mon impossibilité à utiliser mes archives personnelles 
auprès des médias, il me fallait déterminer qu'en faire. La mémoire d'une guerre est-elle envisageable lorsque celle-ci est toujours d'actualité ? Cette option ne me semblait pas non plus appropriée. J'ai finalement décidé d'en faire un livre noir sans texte, comme une boite noire, un album de famille : Souvenirs de Syrie. Ces photographies n'existent donc que dans ce livre.

EG : Ce que tu interroges dans le quotidien des zones de conflits, c'est aussi les moments d'attente, d'ennui, d'angoisse. Tu retranscris ces guerres en effaçant tout effet sensationnel, de sensationnalisme. Dans ta dynamique de comprendre la guerre, dans sa mise en scène, dans sa construction, tu intègres à $360^{\circ}$ le fait d'être devant, derrière, autour, soit l'environnement territorial. Tu parviens ainsi à définir les mécanismes de la guerre mais on perçoit aussi le respect porté envers le combattant. Cela est possible grâce à ta constance sur du long terme, en créant des liens de confiance et amicaux. Sur les dernières minutes, de ton film Quand les nuages parleront, on comprend ce franchissement illégal de la Turquie vers la Syrie. Cet extrait m'a personnellement touché car nous touchons ici le cœur de l'acte. Puis tu rends hommage, par un portrait, à l'un de tes amis qui a disparu en essayant de traverser
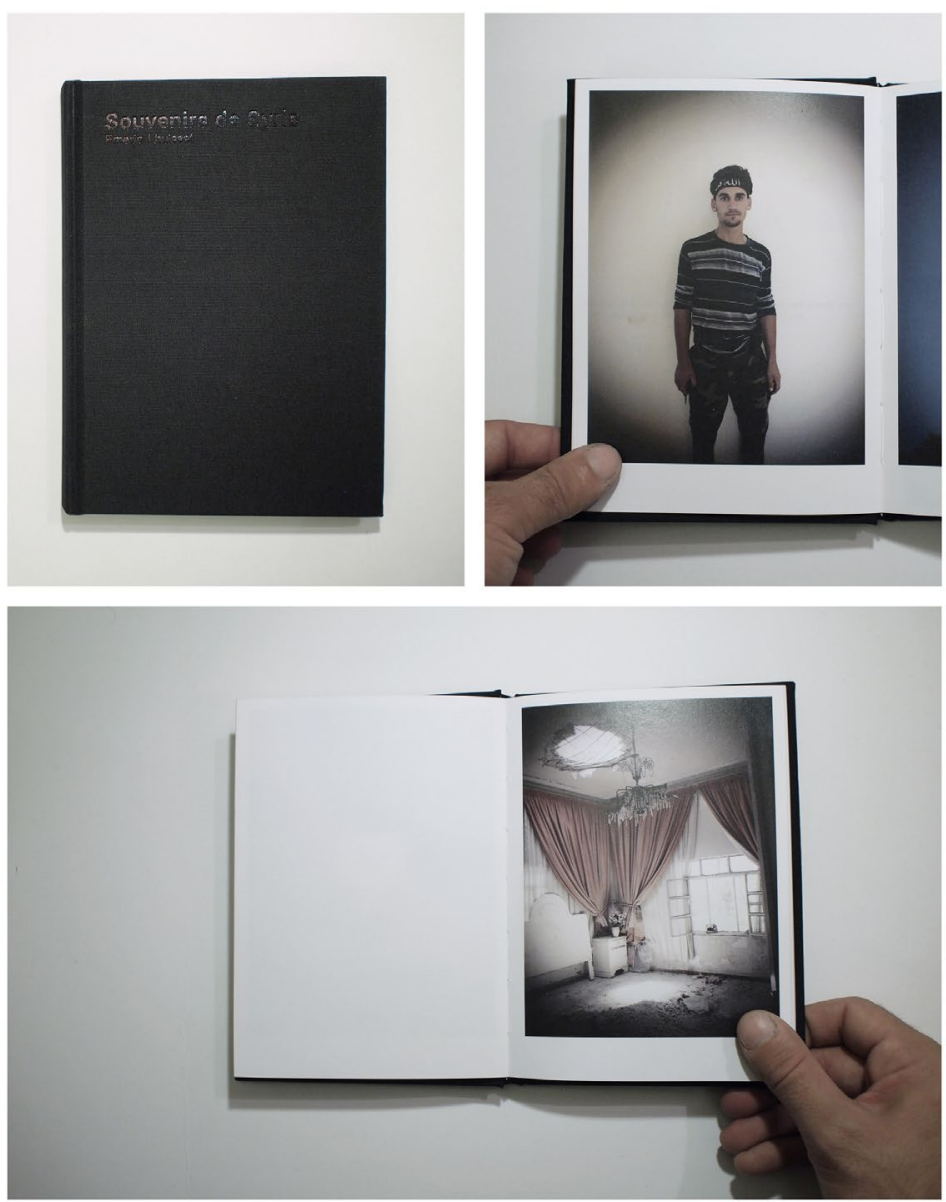

Figure 5. Souvenirs de Syrie, Alep et province d'Idlib (Syrie) juin et août 2012-livre réalisé en 2013. (c) Emeric Lhuisset. Courtoisie de l'artiste. une frontière. Cette expérience dévoilée et sincère est quelque chose que l'on voit rarement dans l'ensemble de la production artistique qui traite, de près ou de loin, nos thématiques.

EL : J'ai choisi de mettre cet extrait de vidéo à la fin de ce film car il fait particulièrement sens avec la question des kurdes. Le territoire kurde est plus vaste que celui que l'Etat turc lui-même. Les kurdes se trouvent dans plusieurs états. C'est ou c'était aussi le cas des Arméniens et des Assyriens dont je parle également. II s'agit de différents peuples existant sur ce vaste territoire mais aussi au-delà des frontières de ce territoire. Finalement ce sont des peuples qui transgressent cette notion de frontière étatique. D'autre part j'ai également voulu terminer avec cette vidéo parce que mon ami Hamidreza est mort sur le tracé d'une frontière, celle qui sépare la Turquie de l'Irak lors d'un bombardement de l'aviation turque. Effectivement, j'ai voulu lui dédier ce film car il m'avait beaucoup aidé notamment pour la série Théâtre de guerre (2011-2012) [Figures 7 \& 8]. II se trouvait que sans comprendre pourquoi je n'avais plus de ses nouvelles jusqu'à ce que des amis m'annoncent qu'il était décédé. Ce franchissement de la frontière reflète aussi cette histoire.

EG : Le concept de frontière est complétement ambivalent. Les géographes eux-mêmes peine à s'accorder sur une même définition. On peut évoquer une ligne, ce qui restreint à l'outil cartographique, originairement militaire. On parle aussi de réseau, de maillage, qui est sans doute plus pertinent lorsque l'on touche le limitrophe.

EL : C'est aussi une zone de tension.

EG : Oui bien sûr. A ce propos, le géographe Claude Raffestin énonce la frontière comme coupure, celle qui contraint l'individu du fait des contrôles, du dispositif et renfort sécuritaire de l'infrastructure. On pense à une coupure, telle une brèche entre deux mondes.

EL : Je n'ai pas théorisé la notion de la frontière mais oui, je pense pouvoir dire que c'est ce qui va séparer. Ce sont des populations, des communautés, qui à travers la création d'un Etat et de frontières, ont décidé de ne pas vivre avec d'autres qui sont à côté. A la base l'idée de frontière est hyper violente, si on l'analyse de ce point de vue-là.

EG : Raffestin dit aussi de la frontière que c'est une couture, quand on envisage les flux et interdépendances sociales, sociétales, économiques...

EL: Une couture ou alors je vois plutôt une suture, car ce sont deux éléments qui ont été 
ensemble puis déchirés, séparés. On crée des ponts à partir de cela.

EG : C'est là aussi une autre image. On parle souvent de Frontières-ponts et de Frontières-murs. Lorsque l'on vit le territoire, on s'en rend bien compte.

EL : Oui, la plaie à soigner d'un côté et la plaie restée ouverte de l'autre. Si on reste dans la métaphore [rires]. En fin de compte, mes expériences avec les frontières ne sont pas très bonnes, mon ressenti est celui d'une vraie tension autour. Car sans même regarder des panneaux les annonçant, on peut détecter lorsque l'on s'en approche. Les militaires sont généralement plus lourdement armés, voire casqués alors qu'ailleurs ils ne le sont pas etc.

EG : Nous-même quand nous étions enfants, avant les accords de Schengen de 1992, avons vécu cette forme de tension au passage de la frontière.

EL : Oui tout à fait. Je me souviens d'anecdotes à ce sujet lorsque je partais en train en Allemagne avec ma grandmère. Elle me rassurait au sujet des douaniers français qui effectivement, en uniformes décontractés, étaient sympas et nous laissaient passer. Elle m'avait averti sur les douaniers allemands qui quant à eux portaient des uniformes stricts et ne nous souriaient pas. J'ignore s'il s'agissait pour elle d'un flashback de la 2nde guerre mondiale ou le fait de montrer nos papiers, mais cette traversée de la frontière la stressait énormément. Aujourd'hui cela nous paraít tellement lointain, voire absurde. Grâce au quasi effacement des frontières en Union Européenne, on est arrivé à quelque chose de très agréable. Mais lors de mon travail en 2016 sur les réfugiés, en passant de l'Allemagne au Danemark, j'avais été choqué par les danois qui avaient posé un checkpoint à la frontière. Afin de de documenter, j'avais pris des photographies avec eux en me faisant passer pour un touriste un peu idiot afin qu'ils ne se méfient pas.

EG : Nous vivons actuellement un repli de la frontière à cause de la pandémie.

EL : Oui, en espérant que ce ne soit qu'une parenthèse.

EG : Par contre, tu te réfères à une méthodologie, celle de l'utilité de jouer au touriste idiot afin de documenter ces situations particulières. Mais peux-tu nous expliquer ton approche lorsque tu te déplaces sur des territoires tels que l'Irak, la Syrie, l'Afghanistan...

EL : Dans ce cas de figure, je fais très peu d'images. Ma démarche est complétement différente dans les
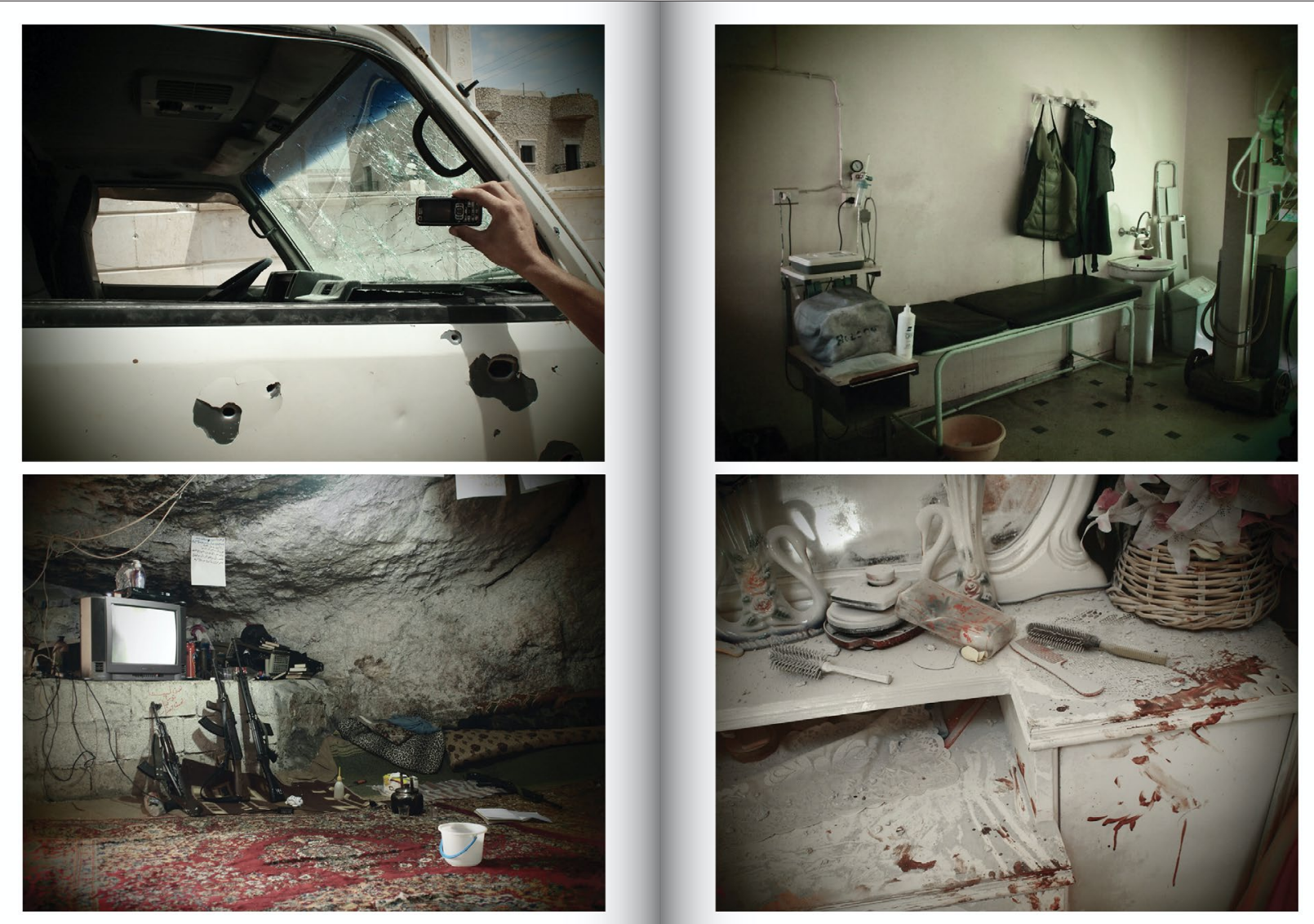

Figure 6. Souvenirs de Syrie, Alep et province d'Idlib (Syrie) juin et août 2012-livre réalisé en 2013. () Emeric Lhuisset. Courtoisie de l'artiste. 
Borders in Globalization Review | Volume 3 | Issue 1 | Fall/Winter 2021

Ganivet, "Frontières \& Mythologies personnelles : Entretien avec Emeric Lhuisset"

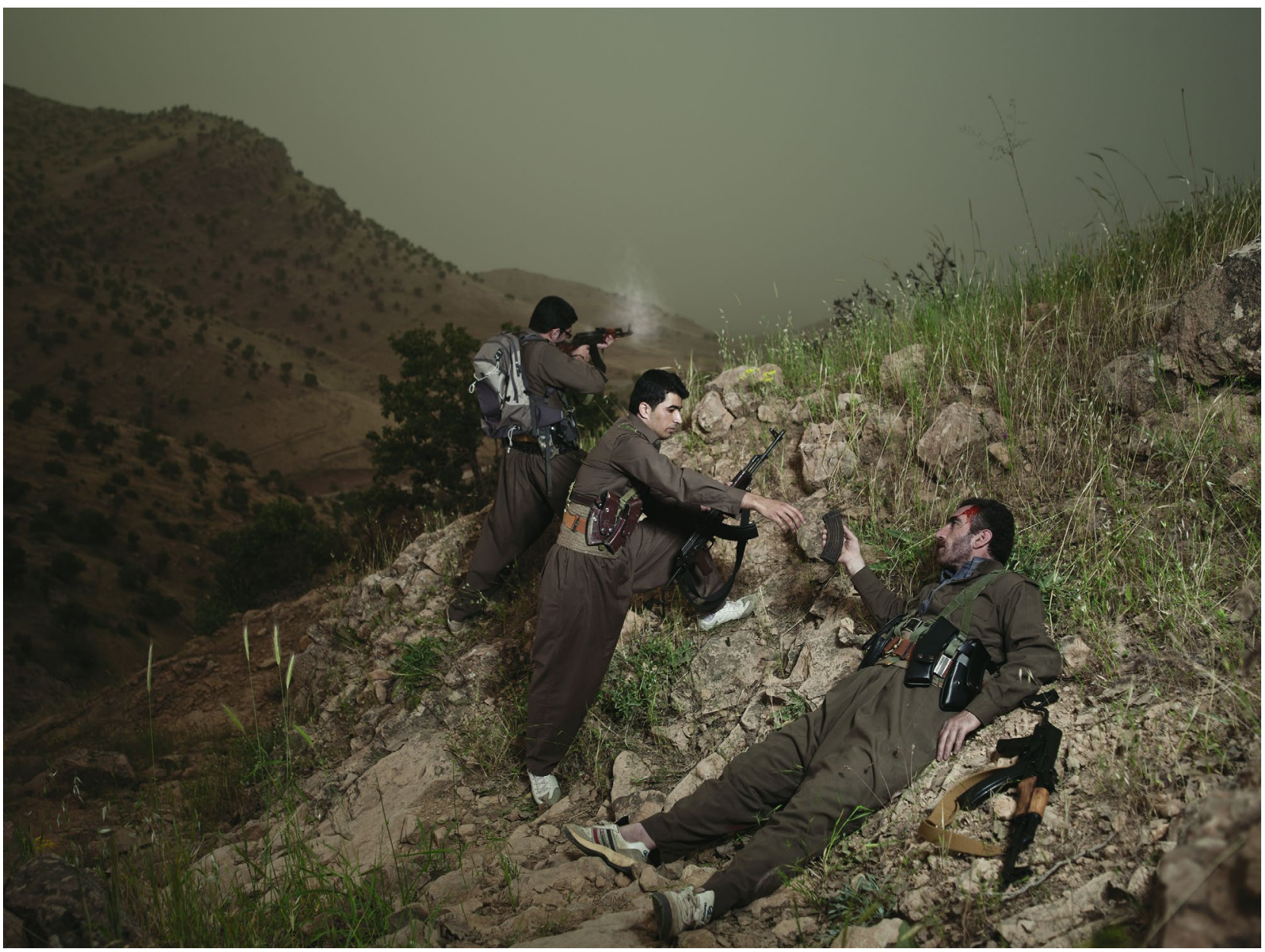

Figure 7. Théâtre de guerre, photographies avec un groupe de guérilla Kurde, Irak, 2011-2012. @ Emeric Lhuisset. Courtoisie de l’artiste.

zones de conflit. Dans un premier temps, je rencontre des personnes et leur parle de mon travail. Cela peut prendre des années pour obtenir ce genre d'images car elles sont basées sur des relations de confiance. II m'a fallu trois ans pour réaliser la série Théâtre de guerre qui comporte quatorze images. Dans la durée, on ne peut pas mentir aux gens. Ils se rendent bien compte de qui tu es. Je n'ai d'ailleurs pas cherché à leur mentir, j'ai juste raconté ce que je faisais, ce qui m'intéressait, ce que je voulais dire. On a parlé, construit ensemble le projet. Et c'est vraiment cette démarche qui m'intéresse. En effet, lorsque j'arrive, c'est avec ma culture, mon bagage, avec tout cette mythologie personnelle comme dirait Roland Barthes. Les personnes que je vais rencontrer ont également la leur. C'est alors que nous confrontons nos regards, non pas dans l'affrontement mais dans l'échange. On va réfléchir et construire ensemble le projet. Mes idées, ma culture ne font pas forcément sens pour eux. Lorsque je fais une œuvre ce n'est pas là-bas pour ici. Cette œuvre doit être aussi pertinente là-bas qu'ici. Pour moi c'est essentiel. C'est la raison pour laquelle j'expose aussi beaucoup au Moyen-Orient. J'essaye de montrer mon travail aussi là-bas et pas juste de le faire là-bas. Je traite de sujets qui me sont assez éloignés parce que j'ai besoin d'avoir assez de distance avec mon sujet de travail pour pouvoir le traiter. II m'est très difficile d'aborder des sujets dont je suis trop proche. Par exemple, j'ai grandi en banlieue parisienne et on m'a souvent demandé pourquoi je ne travaillais pas sur ce sujet. II me manquerait la distance et l'objectivité nécessaires. J'ai ce besoin d'avoir une distance sur mes sujets, qu'ils n'aient au préalable pas de lien direct avec moi-même. C'est un parti pris en somme.

EG : Néanmoins actuellement, avec nos restrictions de voyages dus à la pandémie, tu es en train de développer un projet pour lequel tu iras au domicile des personnes.

EL : Oui mais c'est finalement habituel dans mon travail. Par exemple, lorsque je suis avec des groupes armés, ou avec les réfugiés, on partage une intimité. Dans ce cas précis, j'ai été les retrouver et les ai suivis en tant qu'ami. C'est ce qui m'a permis de faire ce genre d'images. J'ai documenté des moments passés avec des amis.

EG : Mais là c'est en France du coup.

EL : Mon travail avec les réfugiés s'est déroulé dans un premier temps en Allemagne et au Danemark. Puis dans un deuxième temps en France, pour ce qui concerne la 
seconde génération. J'ai travaillé avec Inès dont le père avait quittél'Algérie, avec Sarah dont le père avait quitté l'Irak de Saddam Hussain, tout comme le père de ma cousine, donc mon oncle. Finalement dans ma famille... [rires] Je travaille beaucoup dans et avec l'intimité. J'aime comprendre les gens. L'approche est anthropologique, voire même psychanalytique. C'est tardivement que je me suis rendu compte de cette dimension, celle qui consiste à se pencher sur les gens eux-mêmes, sur ce qu'ils pensent. Pendant toute ma scolarité aux Beaux-Arts, je suivais des cours de psychanalyse de l'art avec Christian Gaillard. D'avoir étudié les écrits de Jung, Freud etc. m'a certainement influencé car instinctivement, je demandais aux gens si telle chose ne leur renvoyait pas à telle autre etc. Sur le terrain, je procède de la même façon. Au début, les groupes de guerilla te sortent un discours calé sur celui de la propagande du groupe. Cela n'a rien de négatif, c'est juste l'image qu'ils souhaitent donner. Pour passer au-delà, la dimension psychanalytique est bienvenue. II s'agit de comprendre l'individu, les raisons qui l'ont amené à arriver à cette situation, vers quoi il souhaite aller. En dehors de tout discours, qu'est-ce qui l'intéresse véritablement ? Et on en revient à : « Ah oui mais quand j'étais petit... ». C'est fascinant car c'est souvent lié.

EG : Si de part et d'autres ta démarche de compréhension de la personne est la même, tu dois aussi trouver des traits psychologiques communs entre chaque combattant.

EL : Oui mieux comprendre le profil des combattants avec qui je vais travailler est une étape fondamentale. On se livre mutuellement, ce qui sur des zones de guerre n'est pas sans risque. Ma vie est un peu entre leurs mains. Lorsque tu te retrouves avec un groupe de guerilla dans une grotte en Syrie, ta vie ne vaut pas chère, ou au contraire elle le vaut. II faut instaurer une confiance mutuelle. Lorsque les combattants me laissent faire des images, ils se donnent à moi aussi. II est fondamental de comprendre les personnes à qui tu te livres. Au sein du groupe, de la communauté, il faut savoir à qui tu peux faire plus ou moins confiance. Comprendre l'ensemble des mécanismes est utile à la fois pour le projet, pour ma propre sécurité, mais aussi simplement par intérêt humain. II marrive fréquemment d'essayer de comprendre des personnes dont l'idéologie est aux antipodes de la mienne. J'essaye de comprendre les mécanismes d'une pensée avec laquelle je ne suis personnellement pas d'accord. En saisissant ses mécanismes, je peux essayer de les déconstruire. Notamment vis-à-vis des regards portés

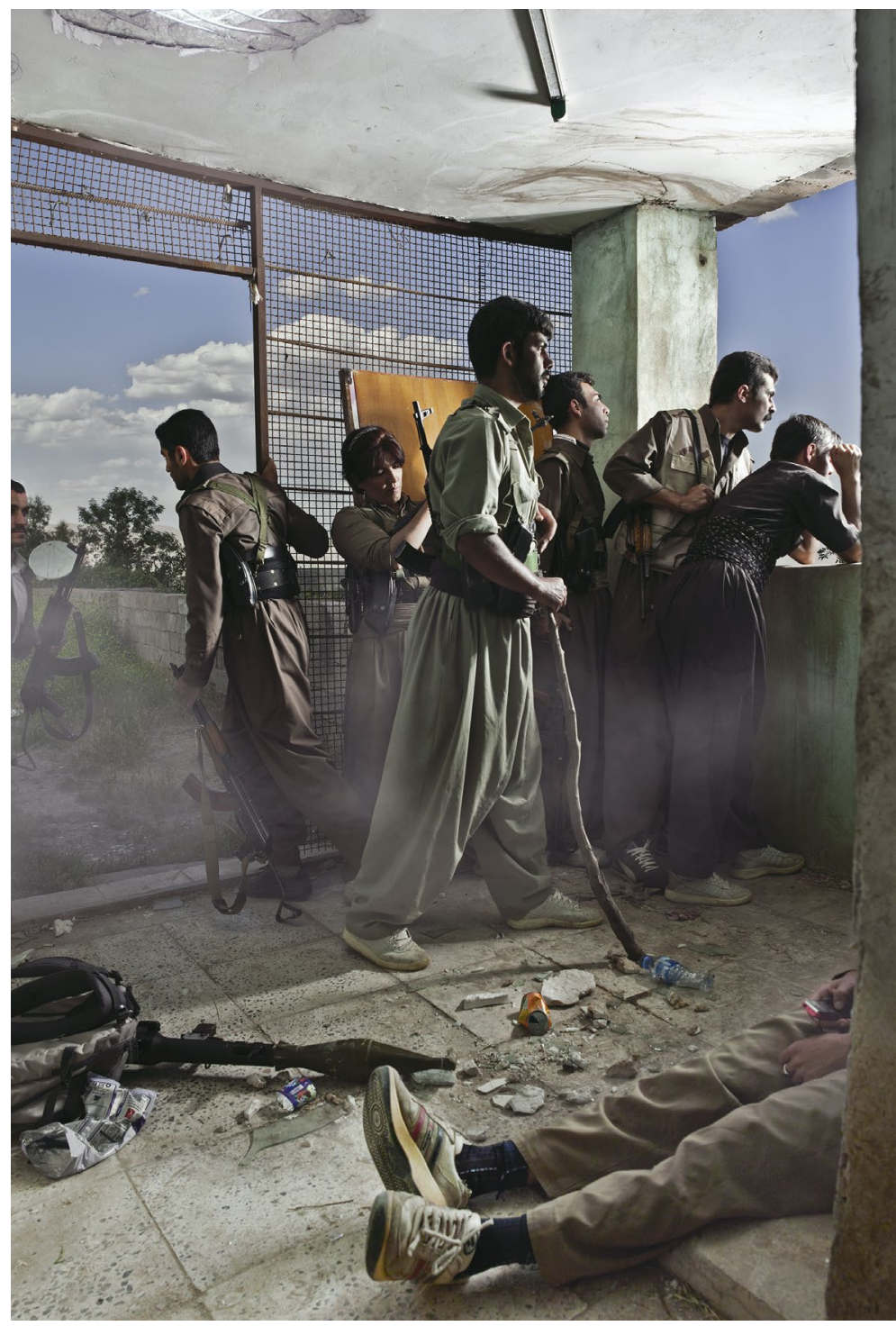

Figure 7. Théâtre de guerre, photographies avec un groupe de guérilla Kurde, Irak, 2011-2012. (c) Emeric Lhuisset. Courtoisie de l'artiste. haineux. Alors qu'en approfondissant, tu t'aperçois qu'il s'agit rarement d'un sentiment de haine, fait d'ailleurs très rare, mais plutôt d'une incompréhension et de la peur. De l'incompréhension qui entraîne de la peur, qui entraîne à son tour un discours très violent. Donc, si on s'arrête au premier regard qui s'apparente à de la haine, la suite de l'approche sera erronée et on ne parviendra pas à déconstruire les regards.

EG : Pourquoi souhaites-tu déconstruire ce type de regards?

EL : Car j'estime que certains d'entre eux sont injustes, qu'ils créent de la violence, un danger à la fois pour les personnes contre lesquelles ils se dressent mais aussi pour la société en son entier. Beaucoup de gens avec ce regard a priori haineux vont le partager, vont mettre en place des leaders qui vont décider en fonction. C'est de cette façon que les sociétés totalitaires, les 
absurdités dangereuses, les massacres, les génocides etc. arrivent. Leur cause revient essentiellement à ce type de mécanisme donc il est pour moi essentiel de déconstruire ce type de regards.

EG : Comment perçois-tu cette crispation, cette montée des populismes, des nationalismes? Je pense en particulier à l'AFD en Allemagne, ce qui est un comble dans le sens où l'on ne pensait pas que cela pouvait arriver dans ce pays.

EL : C'est très inquiétant. C'est la raison pour laquelle il faut d'autant plus essayer de déconstruire les regards. II faut tenter de se dresser contre. Mais pas comme un mur, ce n'est pas la solution. Car les gens finiront par taper dessus et le casser. Donc plutôt s'insinuer et essayer non pas de les convaincre, mais plutôt les inviter à se poser des questions. Par exemple, j'ai mis en parallèle mon travail sur les réfugiés avec celui des combattants kurdes. Il y a entre les deux un écart de huit ans. Ce qui m'intéresse c'est que d'un côté ces combattants sont héroïsés quasiment à l'unanimité en Occident, quelques soit les bords politiques. Les personnes qui sont dans le rejet des réfugiés, idéalisent également ces combattants kurdes : ils sont ceux qui ont combattu Daesh. Sauf que dans mon projet, c'est précisément ces mêmes combattants, ces personnes que tu retrouves huit ans plus tard, devenues des réfugiés. Or il y a tout un pan de la population qui considère les réfugiés comme des parias, des parasites alors même qu'ils héroïsent ces combattants kurdes. Or comment peut-on héroïser une personne et une fois que celle-ci a franchi la mer, la considérer comme un paria, un parasite ? Les gens ne font pas forcément d'emblée ce rapprochement. C'est en le confrontant sur ce point que des doutes, des fissures dans leur rhétorique, leur idéologie, vont se créer. II s'agit de fissurer ces idéologies afin qu'elles ne s'effondrent. C'est tout du moins la manière dont j'essaye de procéder. C'est flagrant lors de présentations publiques de mon travail. A Paris-Photo par exemple, lorsque j'ai dévoilé mon travail sur les réfugiés, certains dans l'assistance n'avaient pas véritablement envie d'écouter. Néanmoins au fur et à mesure, en racontant les histoires individuelles des réfugiés, les yeux de l'auditoire se sont un peu éclairés, comme si quelque chose s'était passé en eux. La visite terminée, ils finissaient par discuter entre eux du sujet, revenaient voir certaines images etc. Ce sont ces mêmes personnes qui au début se disaient qu'elles allaient suivre la visite par politesse. Et c'est donc à ce moment précis que je me dis que cela fonctionne. Les gens ne vont pas forcement changer leur idéologie mais ils vont commencer à s'interroger, peut-être autrement. Et c'est pour moi essentiel.

EG : Alors que ces combattants sont héroïsés, lorsqu'ils arrivent sur un territoire en Occident, en l'occurrence ici en France, c'est précisément la question de l'autre, le rapport à l'Altérité qui survient. On a bien vu les répercussions de la « crise migratoire » en 2015 et encore aujourd'hui, celles qui ont conduit à un repli des frontières. Que faire de cet Autre qui dérange ? Comme tu le précises, la peur et l'incompréhension guident, alors que l'on a besoin de l'autre. L'excuse est souvent au terme démographique, mais le besoin va bien au-delà.

EL : Selon moi c'est une peur qui a été créée par des politiques. II y a un jeu sur la méconnaissance, l'ignorance, afin d'appuyer leur pouvoir. On arrive toujours à cette recherche d'avoir un bouc-émissaire. De tout temps cette recherche apparaît. Ça a été le juif, le protestant, le gitan... celui qu'on ne connait pas ou du moins que l'on connait mal. J'ai beaucoup travaillé sur la Turquie où le pouvoir repose et se construit sur l'idée même d'un ennemi intérieur. Qu'il s'agisse des arméniens, des assyriens, des grecs pontiques, des alévis, des kurdes : il faut toujours construire un ennemi de l'intérieur.

EG : Ce qui est effrayant c'est le relais pris par les nouvelles technologies où on finit par rester dans son propre clan, sans possibilités de traverse. Les passerelles entre les savoirs, les connaissances sont moindre.

EL : Je me pose beaucoup de questions par rapport aux nouvelles technologies. Oui effectivement, ça facilite la communication des idées.

EG : Mais le cadre reste entre nous, nos communautés.

EL : Oui mais n'était-ce pas déjà le cas avant ? J'ai retrouvé par exemple un livre édité en France dans les années vingt : « Le complot juif-allemand ». Ce genre de livres circulait, s'échangeait. Les complots ont toujours existé, avant même l'apport technologique. Elles permettent juste d'accélérer l'information, comme l'a été l'invention de l'imprimerie. Le vrai problème, à mon avis, c'est qu'en fin de compte ceux qui pourraient travailler à la déconstruction des complots n'y parviennent pas assez efficacement. Les médias reconnus regroupent une information accessible mais très tôt il y a eu l'alternative d'internet et des réseaux sociaux. Celle-ci a été très vite accaparée par ceux dont l'information ne pouvait pas passer par les grands médias. La toile leur a servi à diffuser leurs idées, ce qui n'était pas forcément le cas pour la majorité des médias classiques qui n'en avaient pas besoin car ils possédaient déjà leurs canaux de diffusion. Du coup, ils sont arrivés plus tardivement sur un champ déjà investi par les complotistes. Bien sûr poser le doute est assez sain mais le problème est la manière dont on s'interroge. De quelles connaissances sur le sujet dispose-t-on? Qui est le narrateur? Certains complots sont extrêmement complexes à démêler car solidement construits, mélangeant vraies et fausses informations. De plus, le complot peut avoir un effet séduisant dans ce qu'il apporte comme réponse facile. Alors qu'il faudrait aussi savoir accepter de ne pas savoir, on ne peut avoir réponse à tout ! 